\title{
Synthesis of Highly Ordered Si-Containing Fluorinated Block Copolymers
}

\author{
Jianuo Zhou, Xuemiao Li, and Hai Deng* \\ School of Micro-Electronics, State Key Laboratory of Molecular Engineering of Polymers \\ Fudan University, Shanghai 200433, China \\ *haideng@fudan.edu.cn
}

\begin{abstract}
Series of Si-containing, especially polyhedral oligomeric silsesquioxane (POSS)containing fluorinated block copolymers (BCPs), poly(styryl polyhedral oligomeric silsesquioxane)-block-poly(hepatafluorobutyl methacrylate) (PStPOSS- $b$-PHFBMA) were synthesized via living polymerizations. The flory-huggins parameter $\left(\chi\right.$, at $\left.150{ }^{\circ} \mathrm{C}\right)$ of PStPOSS- $b$-PHFBMA BCP was 0.060 . Highly ordered hexagonal domain with $13.2 \mathrm{~nm} d$ spacing was observed by small-angle X-ray scattering (SAXS) after $10 \mathrm{~h} 160{ }^{\circ} \mathrm{C}$ annealing, exhibiting rough line patterns in scanning electron microscope (SEM). $\mathrm{SiO}_{1.5}$ residue (13.7 $\mathrm{wt} \%$ ) still remained after $700{ }^{\circ} \mathrm{C}$ sintering in thermal gravimetric analysis (TGA).
\end{abstract}

Keywords: POSS BCP, Polyhedral oligomeric silsesquioxane, POSS, Fluorinated BCP, DSA, Hexagonal line, Organic-inorganic hybrid

\section{Introduction}

As a potential strategy for next-generation lithography technology (sub-10 nm), Directed SelfAssembly (DSA) with high resolution, has been attracting great attention from both academic and industrial fields. The most common materials used for DSA are block copolymers (BCPs) [1-6]. Extreme Ultraviolet lithography technology (EUVL) [7] has entered high-volume-manufacture (HVM) era with $13 \mathrm{~nm}$ half-pitch resolution, while patterning technologies for sub-10 $\mathrm{nm}$ resolution is still under defined.

Following the strategy of synthesizing BCPs with high flory-huggins parameter $(\chi)$, indicating high incompatibility between two blocks, and low degree of polymerization $(N)$, various BCP systems with sub-10 nm domains were reported [8]. Our group recently reported several series of high $\chi$ fluorinated BCPs which can form 4-8 nm line-width pattern. Besides high resolution, the fluorinated block can drive BCPs to form cylindrical and lamellar line patterns at a very fast speed $(\sim 1 \mathrm{~min})$ at rather low temperature $\left(<100{ }^{\circ} \mathrm{C}\right)$, showing great throughput advantage [9-17].

Besides high resolution and fast self-assembly speed, high etch contrast is also a key property for patterning materials. Si-containing BCPs, especially polyhedral oligomeric silsesquioxane (POSS)-containing BCPs, were widely studied, whose $\chi$ value ranged from 0.03 to 0.45 with max resolution of $4.4 \mathrm{~nm}$ [18] were reported [18-25].

In order to enhance the etch contrast for fast assembling fluorinated BCPs, high etch resistant metal-containing block and components were integrated into the BCPs $[11,26]$. In this study, Sicontaining, especially POSS-containing BCPs, were synthesized and studied. Styryl-POSScontaining fluorinated BCPs, poly(styryl polyhedral oligomeric silsesquioxane)-block-poly(hepatafluorobutyl methacrylate) (PStPOSS- $b$-PHFBMA), and poly(trimethylsilylstyrene)-block-poly(pentadecafluorooctyl methacrylate) (PTMSS- $b$-PPDFMA) BCPs were synthesized via living polymerizations. Preliminary results of $\chi$ value and phase-separated patterns were reported in this paper.

\section{Experimental}

2.1. Synthesis and characterization

A newly-designed styryl polyhedral oligomeric silsesquioxane (StPOSS) monomer was used to synthesize PStPOSS- $b$-PHFBMA BCP via two-step reversible addition-fragmentation chain transfer (RAFT) polymerization. As a comparison, another Si- and F-containing BCP, PTMSS-b-PPDFMA, 


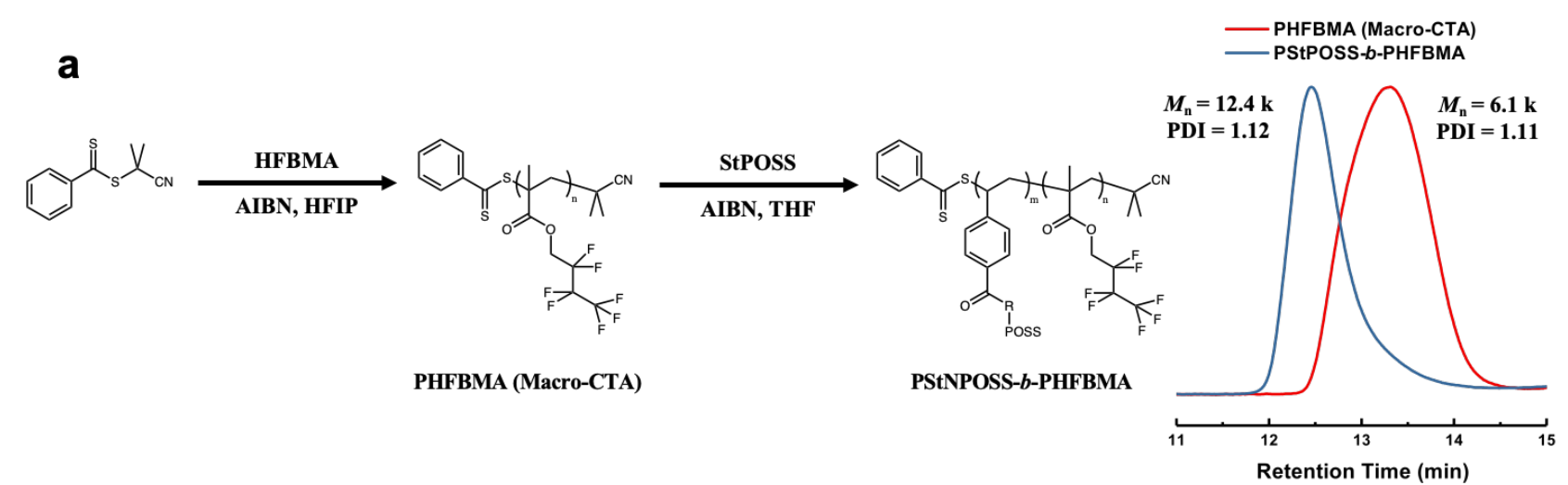

b

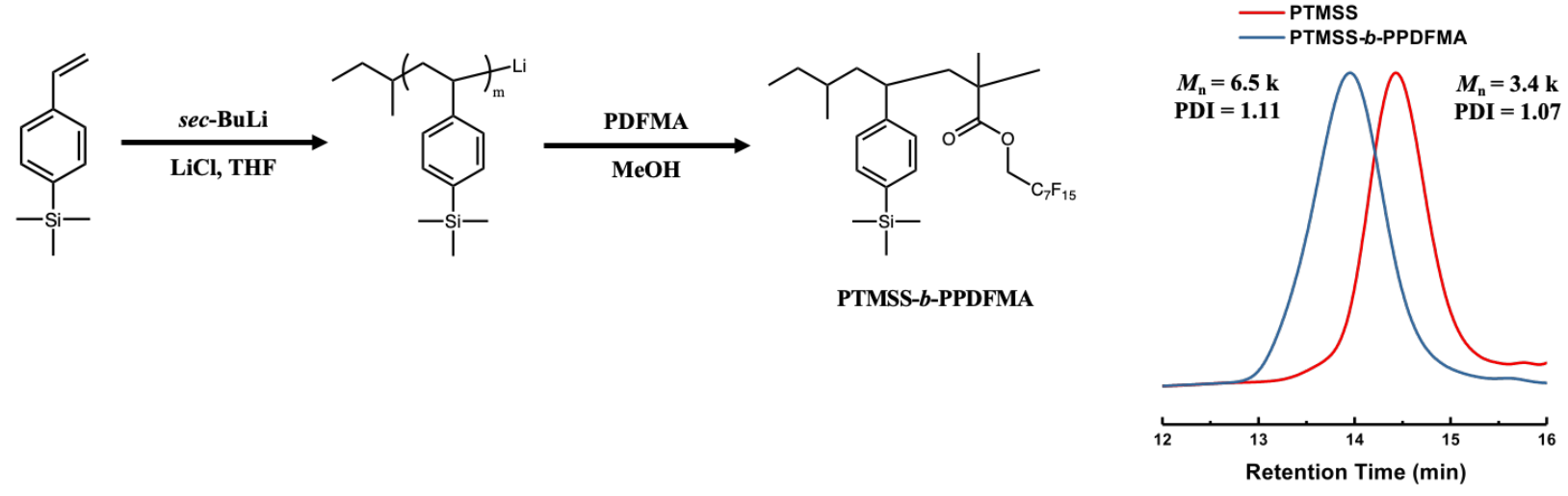

Fig. 1. Synthesis of (a) PStNPOSS- $b$-PHFBMA $\left(M_{\mathrm{n}}=12.4 \mathrm{k}\right.$, PDI $\left.=1.12\right)$ and (b) PTMSS- $b$-PPDFMA $\left(M_{\mathrm{n}}=6.5 \mathrm{k}\right.$, $\mathrm{PDI}=1.11$ ) via living polymerizations, resulting in higher molecular weight $\mathrm{BCPs}$ with narrow polydispersity.

was also synthesized via living anionic polymerization. Trimethylsilylstyrene (TMSS) monomer was synthesized according to reported method [27]. All the other monomers and initiators were purchased from Energy Chemical or J\&K and purified by neutral alumina column or recrystallization to remove inhibitors and impurities. Lithium chloride was obtained from Alfa Aesar. All the solvents (hexafluoroisopropanol (HFIP), tetrahyfrofuran (THF), methanol, propylene glycol monomethyl ether acetate (PGMEA)) were purchased from Titan and used as received.

All the synthesized BCPs were purified by dissolution three times and precipitation, followed by nuclear magnetic resonance (NMR) and gel permeation chromatograph (GPC) characterizations.

${ }^{1} \mathrm{H}$ NMR spectrums were recorded on a Bruker spectrometer (Advanced HD III) using $\mathrm{CDCl}_{3}$ as a solvent with tetramethylsilane as an internal standard. GPC characterizations were conducted by an Agilent/Wyatt 1260 GPC eluting with THF (flow rate of $1 \mathrm{~mL} \mathrm{~min}^{-1}$ ).

Differential scanning calorimetry (DSC) was performed on a TA Instruments Q2000. Measurements were taken from $-20{ }^{\circ} \mathrm{C}$ to $200^{\circ} \mathrm{C}$ at a heating rate of $10^{\circ} \mathrm{C} \mathrm{min}^{-1}$ upon the second heating.
Thermal gravimetric analysis (TGA) was performed on a PE Pyris 1 and measurements were taken under a nitrogen flow with a heating rate of $10^{\circ} \mathrm{C} \mathrm{min}$.

The small-angle X-ray scattering (SAXS) diffraction patterns of powder polymer samples were collected on a Xenocs Xeuss 2.0 scattering system with a Pilatus 3R $200 \mathrm{~K}$-A detector, which is equipped with $\mathrm{Cu} \mathrm{K} \alpha$ radiation wavelength of 1.54 A. Field-emission scanning electron microscopy (FESEM) images were acquired with a Zeiss Ultra 55 and a Zeiss Gemini SEM500 with an in-lens detector operated at $3 \mathrm{kV}$.

\subsection{BCP bulk sample preparation}

For SAXS characterizations, bulk sample of BCPs were prepared as following procedure. $0.5 \mathrm{~mL}$ $10 \mathrm{wt} \%$ BCP solution (in THF) was drop-cast on a clean Si wafer. After solvent evaporating and sample being dried in a vacuum oven, the resulted bulk samples were annealed on a hot plate at $160{ }^{\circ} \mathrm{C}$ for $10 \mathrm{~h}$ and then quenched on a cold plate.

\subsection{BCP thin film preparation}

$1.0 \mathrm{wt} \%$ solution of BCPs in PGMEA or THF was spin coated onto a Si wafer. After annealing 
Table 1. Characterizations of PStPOSS- $b$-PHFBMA block copolymers

\begin{tabular}{ccccccccc}
\hline Sample & $\begin{array}{c}M_{\mathrm{n}, \mathrm{NMR}^{\mathrm{a}}}{ }^{-1} \\
\left(\mathrm{~kg} \mathrm{~mol}^{-1}\right)\end{array}$ & $\mathrm{PDI}^{\mathrm{b})}$ & $\mathrm{DP}_{\text {StPOSS }}{ }^{\mathrm{a})}$ & DP $_{\text {HFBMA }}{ }^{\mathrm{a})}$ & Morphology $^{\mathrm{c})}$ & $d$-spacing & $\chi_{\mathrm{eff}}^{\mathrm{e})}$ & $\chi N^{\mathrm{f})}$ \\
\hline S1 & 14.1 & 1.08 & 6.6 & 27 & DIS & $/$ & 0.060 \\
S2 & 14.6 & 1.07 & 6.3 & 30 & DIS & $/$ & $\left(150^{\circ} \mathrm{C}\right)$ & 8.1 \\
S3 & 23.7 & 1.12 & 12.7 & 40 & HEX & 13.2 & 13.3 \\
\hline
\end{tabular}

a) The molecular weights $\left(M_{\mathrm{n}, \mathrm{NMR}}\right)$ and degrees of polymerization were calculated by ${ }^{1} \mathrm{H}$ NMR results. b) Polydispersity indexes (PDI) were measured by GPC in THF against PS standards. c) HEX represented hexagonal morphology, while DIS represented disordered structure was obtained after thermal annealing. $d) d$-Spacing represented the domain spacing of block copolymer and was calculated by the equation as $d=2 \pi / \mathrm{q}^{*}$, while $\mathrm{q}^{*}$ was the value of the first order peak in SAXS.e) The effective $\chi$ value at certain temperature. f) $N$ represented the overall degree of polymerization and was calculated by reference volume $v_{0}=118 \AA^{3}$, based on the densities of PStPOSS and PHFBMA. The $N$ values of S1-S3 were 130,135 and 222, respectively.

process, reactive ion etching (RIE) was conducted in a plasma cleaner (Oxford Plasmalab System100) to enhance the image contrast between two blocks under FESEM measurement.

\section{Results and discussion}

3.1. Synthesis of PStPOSS- $b$-PHFBMA and PTMSS- $b$-PPDFMA BCPs

Styryl-POSS-containing fluorinated BCP, PStPOSS- $b$-PHFBMA, and PTMSS- $b$-PPDFMA $\mathrm{BCP}$ were synthesized via living polymerizations. The synthetic routes and GPC curves of PStPOSS$b$-PHFBMA and PTMSS- $b$-PPDFMA were presented in Fig 1. The GPC curves completely shifted to higher molecular weight, indicating both Si-containing BCPs with $\mathrm{PDI}<1.12$ were obtained.

Table 1 summarized molecular weight $\left(M_{\mathrm{n}}\right), \chi$ value and morphology data. The $M_{\mathrm{n}}$ and degree of polymerization (DP) of PStPOSS- $b$-PHFBMA were measured by ${ }^{1} \mathrm{H}$ NMR. Resulted PStPOSS- $b$ PHFBMA BCPs have various $M_{\mathrm{n}}$, ranged from 14.1 $\mathrm{kg} \mathrm{mol}^{-1}$ to $23.7 \mathrm{~kg} \mathrm{~mol}^{-1}$, with POSS block and fluorine methacrylate block, which also are socalled organic-inorganic hybrid material.

\section{$3.2 \chi$ value characterization}

Morphologies and domain spacings of PStPOSS$b$-PHFBMA BCPs were determined by SAXS. All the $\mathrm{BCP}$ bulk samples were thermally annealed at $160{ }^{\circ} \mathrm{C}$ for $10 \mathrm{~h}$ to achieve equilibrium microphase separation state. As shown in Table 1, S3 could form highly ordered $13.2 \mathrm{~nm}$ hexagonal domain after thermal annealing, while S1 and S2 obtained disordered structure.

The effective $\chi$ value at $150{ }^{\circ} \mathrm{C}$ of PStPOSS- $b$ PHFBMA was calculated to be 0.060 , higher than conventional PS- $b$-PMMA BCP $(\chi=0.030$, at 150 $\left.{ }^{\circ} \mathrm{C}\right)$ [28], but lower than reported POSS-containing BCPs [24]. The $\chi \mathrm{N}$ value of $\mathrm{S} 1$ and $\mathrm{S} 2 \mathrm{BCP}$ was lower than the critical value of $\chi \mathrm{N}=10.5$ [8],
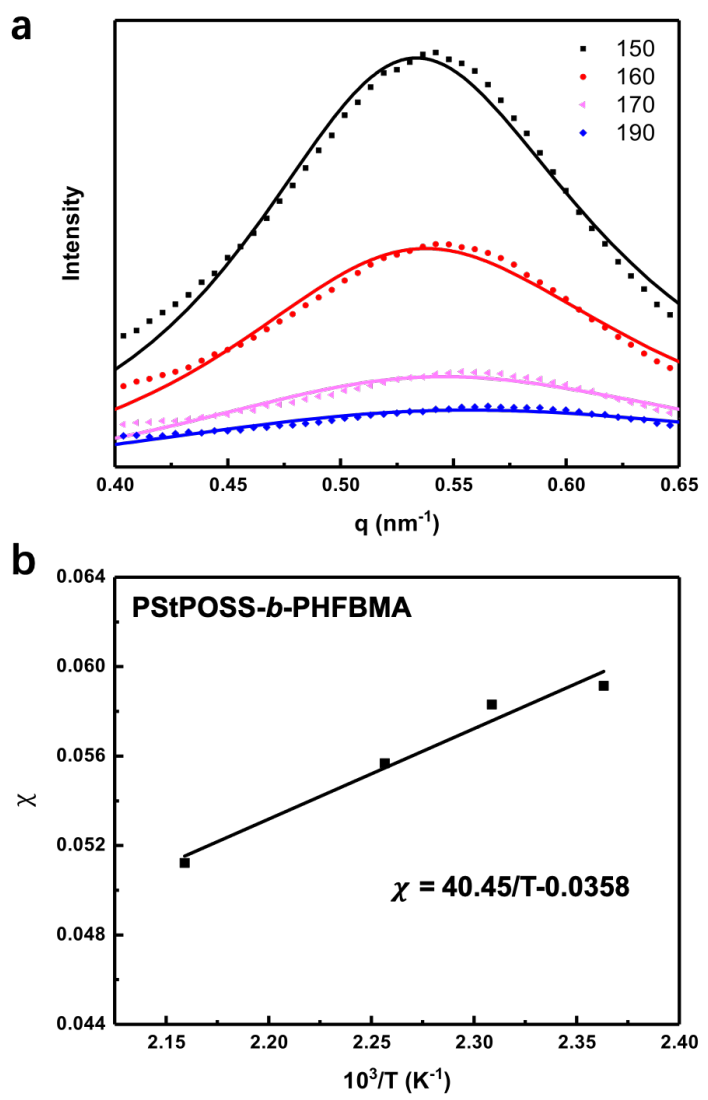

Fig. 2. (a) Fitlines of SAXS intensities of disordered PStPOSS- $b$-PHFBMA at various temperatures. (b) Temperature dependence of $\chi$ between PStPOSS block and PHFBMA block.

resulting that $\mathrm{S} 1$ and $\mathrm{S} 2$ could not obtain ordered structure.

The $\chi$ value of PStPOSS- $b$-PHFBMA was estimated by Leibler's mean field theory using the random phase approximation of the absolute intensity from SAXS (Fig. 2a) [29-31]. Fully disordered BCPs were used to estimate $\chi$ values. The $\chi$ value of PStPOSS- $b$-PHFBMA BCP has a temperature dependence as $\chi=40.45 / \mathrm{T}-0.0358$ (Fig 2b). 

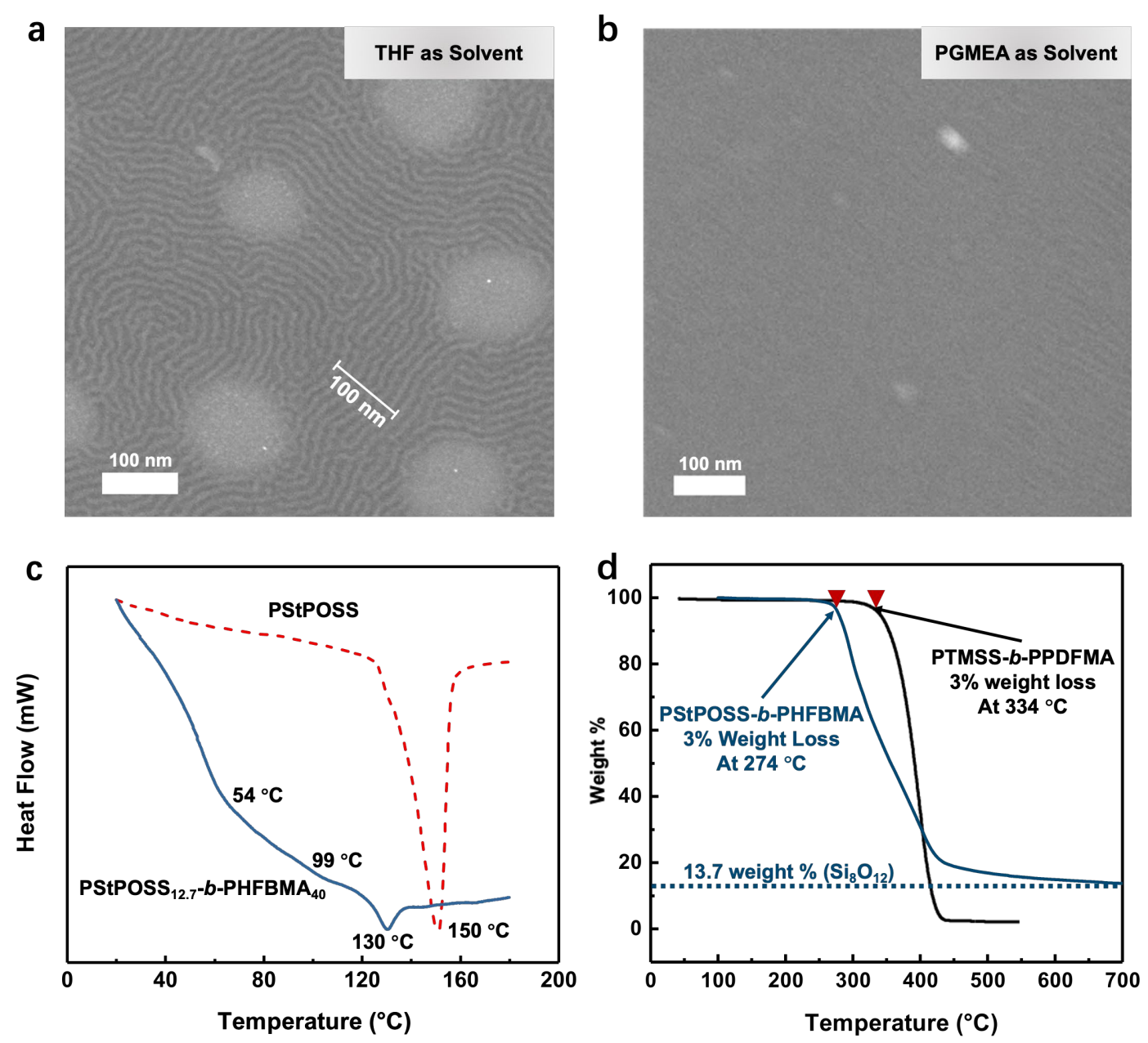

Fig. 3. The FESEM images of PStPOSS- $b$-PHFBMA (S3) thin film prepared by (a) THF and (b) PGMEA. The dark and bright lines correspond to PStNPOSS and PHFBMA microdomains, respectively. (c) DSC curves of PStPOSS- $b$ PHFBMA $\left(\mathrm{T}_{\mathrm{g}}, 54{ }^{\circ} \mathrm{C}\right.$ and $\left.99{ }^{\circ} \mathrm{C} ; \mathrm{T}_{\mathrm{m}}, 130{ }^{\circ} \mathrm{C}\right)$ compared to PStPOSS $\left(\mathrm{T}_{\mathrm{m}}, 150{ }^{\circ} \mathrm{C}\right)$ homopolymer. (d) TGA curves of PStPOSS- $b$-PHFBMA $\left(\mathrm{S} 3, \mathrm{~T}_{\mathrm{d}}, 274^{\circ} \mathrm{C}\right)$ compared to PTMSS- $b$-PPDFMA $\left(\mathrm{T}_{\mathrm{d}}, 334^{\circ} \mathrm{C}\right)$. Residue of PStPOSS- $b$-PHFBMA was $13.7 \mathrm{wt} \%$ at $700{ }^{\circ} \mathrm{C}$, versus $2 \mathrm{wt} \%$ of PTMSS-BCP at $430{ }^{\circ} \mathrm{C}$.

\subsection{Phase separation in thin film}

As well known, POSS monomer is crystallizable and can form crystalline domain when served as pendant group covalently linked to polymer backbone $[32,33]$. The nanostructure of PStPOSS$b$-PHFBMA (S3) thin films prepared by various solvents, PGMEA and THF, were characterized by FESEM after $\mathrm{CF}_{4}$ RIE treatment.

Distinct $7.7 \mathrm{~nm}$ line-pattern was observed in the thin film prepared by THF compared to thin film prepared by PGMEA (Fig. 3a-b). However, the linepattern in THF-prepared thin film still had rather high roughness which might be caused by POSS crystalline.

3.4 Themal analysis of organic-inorganic hybrid BCP (PStPOSS- $b$-PHFBMA)

As the DSC curves presented in Fig. $3 c$, the $T_{m}$ of
PStPOSS homopolymer and PStPOSS- $b$-PHFBMA were $150{ }^{\circ} \mathrm{C}$ and $130{ }^{\circ} \mathrm{C}$, respectively. The POSS crystalline could retard the polymer chain mobility $[32,33]$, resulting in relatively rough line-pattern. PStPOSS- $b$-PHFBMA (S3) has two glass transition temperature $\left(\mathrm{T}_{\mathrm{g}}\right)$, similar to previously reported PSb-PPDFMA BCP [9]. The $\mathrm{T}_{\mathrm{g}}$ at $54{ }^{\circ} \mathrm{C}$ corresponded to PHFBMA, while $\mathrm{T}_{\mathrm{g}}$ at $99{ }^{\circ} \mathrm{C}$ was assigned to PStNPOSS block.

The thermal stability of PStPOSS- $b$-PHFBMA (S3) was further investigated by TGA comparing to PTMSS- $b$-PPDFMA (Fig. 3d). The decomposition temperatures $\left(\mathrm{T}_{\mathrm{d}}\right)$ of PStPOSS- $b$-PHFBMA and PTMSS- $b$-PPDFMA were $274{ }^{\circ} \mathrm{C}$ and $334{ }^{\circ} \mathrm{C}$, respectively. As PStPOSS- $b$-PHFBMA contained rigid inorganic POSS cage similar to $\mathrm{SiO}_{2}$, the inorganic residue (13.7 wt $\%)$ of PStPOSS- $b$ PHFBMA was consistent with theoretical fraction of $\mathrm{Si}_{8} \mathrm{O}_{12}\left(13.0 \mathrm{wt} \%\right.$ ) after $700{ }^{\circ} \mathrm{C}$ sintering (Fig 3d), 
indicating inorganic characteristics. Comparing to POSS BCPs, Si in PTMSS- $b$-PPDFMA almost vanished after heating up to $450{ }^{\circ} \mathrm{C}$, similar to normal organic compound.

\section{Conclusion}

Series of POSS-containing fluorinated BCPs, PStPOSS- $b$-PHFBMA, were synthesized via RAFT living polymerization. Resulted BCPs showed higher molecular weight compared to PHFBMA marco-CTA, and the polydispersity was lower than 1.15 , which indicated PStPOSS- $b$-PHFBMA BCP was obtained. The effective $\chi$ value $\left(150^{\circ} \mathrm{C}\right)$ of our BCPs is 0.060 , lower than previously reported by others. Ordered hexagonal domain with $13.2 \mathrm{~nm} d$ spacing was observed once $\chi \mathrm{N}>10.5$. The SEM image further confirmed the highly ordered linepattern. Due to the POSS crystalline effect showed in DSC, the lines were quite rough. Benefit from the inorganic POSS cage, 13.7 wt $\%$ of PStPOSS- $b$ PHFBMA BCP still remained at $700{ }^{\circ} \mathrm{C}$, which is consistent with the theoretical inorganic composition of $\mathrm{Si}_{8} \mathrm{O}_{12}$.

\section{Acknowledgement}

This work was supported financially from the Shanghai Science and Technology Committee (18511104900) and Shanghai TD Advanced Material Technology Inc. (KCH1717170). The authors also acknowledge experimental support from the State Key Laboratory of Molecular Engineering of Polymers and the Nano-fabrication Laboratory of Fudan University.

\section{References}

1. R. Ruiz, H. Kang, F. A. Detcheverry, E. Dobisz, D. S. Kercher, T. R. Albrecht, J. J. de Pablo, and P. F. Nealey, Science, 321 (2008) 936.

2. M. Li, and C. K. Ober, Materials Today, 9 (2006) 30.

3. J. Bang, U. Jeong, D. Y. Ryu, T. P. Russell, and C. J. Hawker, Adv. Mater, 21(2009) 4769.

4. H.-C. Kim, S.-M. Park, and W. D. Hinsberg, Chem. Rev., 110 (2010) 146.

5. C. M. Bates, M. J. Maher, D. W. Janes, C. J. Ellison, and C. G. Willson, Macromolecules, 47 (2014) 2.

6. C. M. Bates, and F. S. Bates, Macromolecules, 50 (2017) 3.

7. C. Wagner, and N. Harned, Nat. Photonics, 4 (2010) 24.

8. C. Sinturel, F. S. Bates and M. A. Hillmyer, Acs Macro Lett., 4 (2015) 1044.

9. X. Li, J. Li, C. Wang, Y. Liu, and H. Deng, J.
Mater. Chem. C, 7 (2019) 2535.

10. C. Wang, X. Li, and H. Deng, ACS Macro Lett., 8 (2019) 368.

11. X. Li, and H. Deng, Acs Appl. Polym. Mater., 2 (2020) 3601.

12. H. Cao, L. Dai, Y. Liu, X. Li, Z. Yang, and H. Deng, Macromolecules, 53 (2020) 8757.

13. H. Cao, X. Li, Y. Liu, and H. Deng, J. Photopolym. Sci. Technol., 32 (2019) 413.

14. L. Dai, H. Cao, and H. Deng, J. Photopolym. Sci. Technol., 33 (2020) 541.

15. H. Deng, X. Li, Y. Peng, and J. Zhou, Proc. SPIE, 10586 (2018) 105861E.

16. X. Li, Z. Li, L. Dai, H. Cao, and H. Deng, Proc. SPIE, 11326 (2020) 113261E.

17. H. Deng, X. Li, and J. Li, CN107245133B, US20180208697-A1, 2020.

18. Y. D. Luo, D. Montarnal, S. Kirn, W. C. Shi, K. P. Barteau, C. W. Pester, P. D. Hustad, M. D. Christianson, G. H. Fredrickson, E. J. Kramer, and C. J. Hawker, Macromolecules, 48 (2015) 3422 .

19. K. Azuma, J. Sung, Y. Choo, Y. Rokhlenko, J. H. Dwyer, B. Schweitzer, T. Hayakawa, C. O. Osuji, and P. Gopalan, Macromolecules, 51 (2018) 6460.

20. K. Aissou, M. Mumtaz, G. Fleury, G. Portale, C. Navarro, E. Cloutet, C. Brochon, C. A. Ross, and G. Hadziioannou, Adv. Mater., 27 (2015) 261.

21. W. J. Durand, G. Blachut, M. J. Maher, S. Sirard, S. Tein, M. C. Carlson, Y. Asano, S. X. Zhou, A. P. Lane, C. M. Bates, C. J. Ellison, and C. G. Willson, J. Polym. Sci. Part a-Polym. Chem., 53 (2015) 344.

22. T. Hirai, M. Leolukman, T. Hayakawa, M.-A. Kakimoto, and P. Gopalan, Macromolecules, 41 (2008) 4558.

23. T. Hirai, M. Leolukman, C. C. Liu, E. Han, Y. J. Kim, Y. Ishida, T. Hayakawa, M.-A. Kakimoto, P. F. Nealey, and P. Gopalan, $A d v$. Mater., 21 (2009) 4334.

24. R. Nakatani, H. Takano, A. Chandra, Y. Yoshimura, L. Wang, Y. Suzuki, Y. Tanaka, R. Maeda, N. Kihara, S. Minegishi, K. Miyagi, Y. Kasahara, H. Sato, Y. Seino, T. Azuma, H. Yokoyama, C. K. Ober, and T. Hayakawa, ACS Appl. Mater. Interfaces, 9 (2017) 31266.

25. T.-Y. Lo, M. R. Krishnan, K.-Y. Lu, and R.-M. Ho, Progress in Polym. Sci., 77 (2018) 19.

26. G. Wu, and H. Deng, J. Photopolym. Sci. Technol., 33 (2020) 537.

27. S. Langle, F. David-Quillot, A. Balland, M. 
Abarbri, and A. Duchene, J. Organomet. Chem., 671 (2003) 113.

28. T. P. Russell, R. P. Hjelm and P. A. Seeger, Macromolecules, 23 (1990) 890.

29. L. Leibler, Macromolecules, 13 (1980) 1602.

30. Y. Zhao, E. Sivaniah, and T. Hashimoto, Macromolecules, 41 (2008) 9948.
31. A. N. Semenov, Macromolecules, 26 (1993) 6617.

32. J. Waddon, L. Zheng, R. J. Farris, and E. B. Coughlin, Nano Lett., 2 (2002) 1149.

33. A. Lee, and J. D. Lichtenhan, Macromolecules, 31 (1998) 4970. 